\title{
2482. Study on a novel fault diagnosis method based on integrating EMD, fuzzy entropy, improved PSO and SVM
}

\author{
Wu Deng', Rui Yao ${ }^{2}$, Meng Sun ${ }^{3}$, Huimin Zhao ${ }^{4}$, Yinglian Luo ${ }^{5}$, Chang Dong ${ }^{6}$ \\ 1,3,4, 5, ${ }^{6}$ Software Institute, Dalian Jiaotong University, Dalian 116028, China \\ ${ }^{1,2}$ School of Electronics and Information Engineering, Dalian Jiaotong University, Dalian 116028, China \\ $1,3,4,5$ Sichuan Provincial Key Laboratory of Process Equipment and Control \\ (Sichuan University of Science and Engineering), Zigong 64300, China \\ $1,3,4,5$ The State Key Laboratory of Mechanical Transmissions, Chongqing University, \\ Chongqing 400044, China \\ $1,4,5$ Traction Power State Key Laboratory of Southwest Jiaotong University, Chengdu 610031, China \\ ${ }^{1,4}$ Dalian Key Laboratory of Welded Structures and Its Intelligent Manufacturing Technology (IMT) of \\ Rail Transportation Equipment, Dalian Jiaotong University, Dalian 116028, China \\ ${ }^{4}$ Corresponding author \\ E-mail:11w7689@163.com, ${ }^{2} 326358268 @ q q . c o m,{ }^{3} 133321239 @ q q . c o m,{ }^{4} h m \_z h a o 1977 @ 126 . c o m$, \\ 51225378180@qq.com,6815763951@qq.com
}

Received 26 November 2016; received in revised form 24 March 2017; accepted 27 March 2017 DOI https://doi.org/10.21595/jve.2017.18052

Check for updates

\begin{abstract}
In order to effectively improve the fault diagnosis accuracy of motor bearing, a new fault diagnosis method based on integrating empirical mode decomposition(EMD), fuzzy entropy, improved particle swarm optimization(PSO) algorithm and support vector machine (SVM) is proposed in this paper. In the proposed fault diagnosis method, the EMD method is used to decompose vibration signals into a series of basic intrinsic mode functions (IMFs). Then the fuzzy entropy is used to effectively extract the features of vibration signal, which are regarded as input vectors of SVM. The dynamic adjustment strategy of arctangent function of learning factor, decreasing inertia weight of function and adaptive mutation strategy of particles are used to improve the basic PSO algorithm in order to avoid premature convergence, escape from falling into the local optimal value and improve the optimization performance. And the improved PSO algorithms are selected to optimize the parameters of SVM in order to improve the generalization ability and the classification accuracy. And then a new fault diagnosis method is obtained. Finally, the actual vibration signals of motor bearing are selected to verify the effectiveness of the proposed fault diagnosis method. The experiment results show that the improved PSO algorithm can effectively obtain the optimal combination values of parameters of SVM, and the proposed fault diagnosis method can accurately and quickly diagnose the faults of motor bearing with the higher reliability. And it provides a new idea based on making full use of the advantages of each method for studying motor fault diagnosis.
\end{abstract}

Keywords: motor bearing, fault diagnosis, EMD, fuzzy entropy, improved PSO, SVM, parameter optimization.

\section{Introduction}

Bearing is one of the key parts of the motor. Its operation state directly determines the performance of the whole machine. According to the statistics, the motor damage caused by bearing fault is about $40 \%[1,2]$. Bearing fault includes the inner ring fault, the outer ring fault and the rolling element fault. Because the motor is prone to sudden impulse in the operation, it will affect the product quality, cause the accidents, and lead to a serious harm and significant economic losses [3]. So, it is very important to study different fault diagnosis method to accurately identify the motor fault.

In recent years, a lot of fault diagnosis methods have been proposed to detect the faults in order to keep machinery performing at its best state, avoid abnormal event progression and reduce product loss. Chen et al. [4] proposed a new approach based on multi-objective optimization and 
genetic algorithms to diagnose the incipient faults. Wang et al. [5] proposed a fault diagnosis method based on quantum ant colony optimization algorithm and support vector machine. Zhang et al. [6] proposed a fault diagnosis method based on SVM with parameter optimization by ant colony algorithm to obtain a desirable fault diagnosis result. Zhang et al. [7] proposed a novel scheme based on lifting wavelet packet transform, sample entropy, support vector machines and genetic algorithms for bearing fault diagnosis. Zhao et al. [8] proposed a fault diagnosis method based on chaos particle swarm optimization algorithm and support vector machine for sensor. Azade et al. [9] proposed a unique flexible algorithm based on SVM hyper-parameters optimization and artificial neural networks for classifying the condition of centrifugal pump. Du et al. [10] proposed a novel fault diagnosis method based on relevance vector machines with particle swarm optimization algorithm for plunger pump in truck crane. Zhu et al. [11] proposed a fault feature extraction method based on hierarchical entropy for the non-linear dynamic characteristics of roller bearing faulty signals. Unal et al. [12] proposed a fault diagnosis method of rolling bearings by using a genetic algorithm and neural network. Ao et al. [13] proposed a new artificial chemical reaction optimization algorithm o optimize SVM parameters for diagnosing the roller bearing faults. Zhang et al. [14] proposed an intelligent fault diagnosis method of rotating machinery by using support vector machine with ant colony algorithm for synchronous feature selection and parameter optimization. Yang et al. [15] proposed a gear fault diagnosis method based on SVM and ABC algorithm. Chu et al. [16] proposed a FOA-SVM model based on fruit fly optimization algorithm (FOA) and support vector machine (SVM). Duan et al. [17] proposed a new support vector data description method for machinery fault diagnosis with unbalanced datasets. Su et al. [18] proposed a multi-fault diagnosis method based on orthogonal supervised linear local tangent space alignment and LS-SVM for rotating machinery to improve the accuracy of fault diagnosis. Zhang et al. [19] proposed a new hybrid method to optimize the parameters of SVM.

Although these proposed methods have achieved good results in feature extraction and fault diagnosis for rotating machinery, they have their own limitations in the actual application, such as larger calculation and lower accuracy and so on. Motor bearing fault diagnosis generally consists of vibration signal acquisition, fault feature extraction and fault pattern recognition. And the fault feature extraction and fault pattern recognition are two key steps in the fault diagnosis method. The EMD method is a typical signal processing method, which can adaptively decompose the signal into a series of different frequency range and the energy distribution of IMFs. Fuzzy entropy technology introduces the fuzzy entropy set theory. Namely, the similarity measure is fuzzed by selecting exponential function in order to assure that the fuzzy entropy can steadily change according to the parameters. And the fuzzy entropy, sample entropy and approximate entropy have the same nature. For these pattern recognition and diagnosis methods, such as expert system, genetic algorithm, neural network and so on, they are difficult to meet the fault diagnosis and identification with small samples. The SVM is a new machine learning method based on statistics theory. It shows unique advantage and good application prospect in solving small sample problem, and takes on good generalization ability. But the selection result of parameters for SVM will affect the final diagnosis accuracy. The PSO algorithm is a kind of stochastic, parallel optimization technology, which has the characteristics of differentiability, continuation and global optimization capability. Therefore, in order to realize the intelligent diagnosis of motor bearing, the EMD, fuzzy entropy, improved PSO and SVM are introduced into the fault diagnosis to propose a novel fault diagnosis method. The EMD and fuzzy entropy are used to extract the feature of vibration signal, then the dynamic adjustment strategy of learning factor, decreasing inertia weight of function and adaptive mutation strategy of particles are used to improve the PSO algorithm, which is used to optimize the parameters of penalty function and kernel function of SVM in order to obtain an effective fault diagnosis model with the higher accuracy. The validity of the proposed diagnosis method is verified by the actual vibration signal of motor bearing. 


\section{Basic methods}

\subsection{PSO}

The PSO algorithm [20] is a population-based search algorithm based on the simulation of the social behavior of birds within a flock. In PSO algorithm, the particles' positions within the search space are changed based on the social-psychological tendency of individuals in order to delete the success of other individuals. The changing of one particle within the swarm is influenced by the experience or knowledge. The consequence of modeling for this social behavior is that the search is processed in order to return toward previously successful regions in the search space. Namely, the velocity $(v)$ and position $(x)$ of each particle will be changed by the particle best value $(p B)$ and global best value $(g B)$ according to the expressions:

$$
\begin{aligned}
& v_{i_{j}}(t+1)=w v_{i j}(t)+c_{1} r_{1}\left(p B_{i j}(t)-x_{i j}(t)\right)+c_{2} r_{2}\left(g B_{i j}(t)-x_{i j}(t)\right), \\
& x_{i j}(t+1)=x_{i j}(t)+v_{i j}(t+1)
\end{aligned}
$$

where $v_{i j}(t+1)$ is the velocity of particle $i$ at iteration $j, x_{i j}(t+1)$ is the position of particle $i$ th at iteration $j$ th. $w$ is inertia weight to be employed to control the impact of the previous history of velocity. $t$ denotes the iteration number, $c_{1}$ is the cognition learning factor, $c_{2}$ is the social learning factor, $r_{1}$ and $r_{2}$ are random numbers uniformly distributed in $[0,1]$.

The basic flow of the PSO algorithm is shown in Fig. 1.

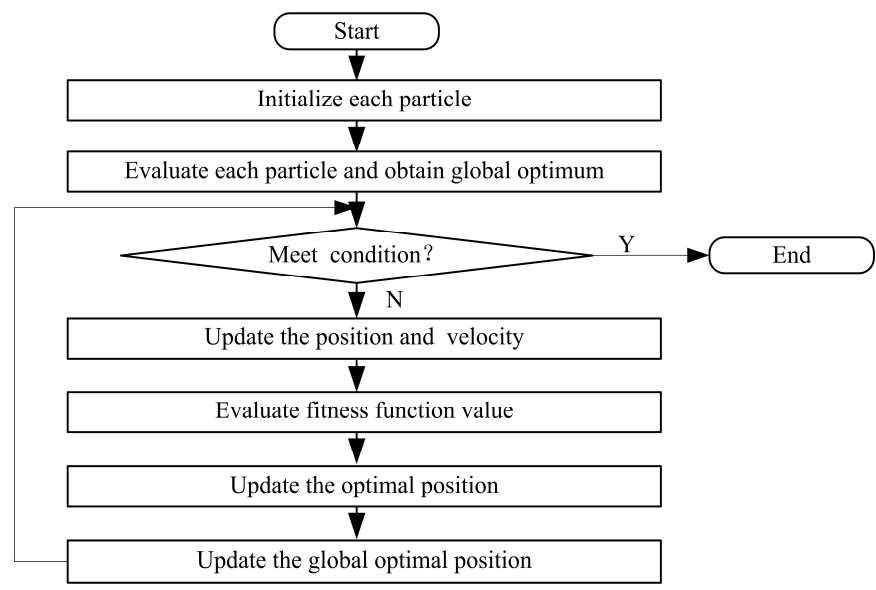

Fig. 1. The basic flow of the PSO algorithm

\subsection{EMD}

The EMD [21] is adaptive decomposition technique. It is based on the direct extraction of the energy associated with various intrinsic time scales in order to generate a collection of intrinsic mode functions (IMFs). The EMD can decompose the complicated signal into a definite number of high-frequency and low frequency components. The sifting process decomposes the original signal $(S(x))$ into a number of IMFs:

$S(t)=\sum_{i=1}^{n} c_{i}(t)+r_{n}(t)$

where $r_{n}(t)$ is residual error function, and represents average trend of signals. IMF components $c_{1}, c_{2}, c_{3}, \ldots, c_{n}$ contain different elements respectively from low to high frequency of signals. 


\subsection{Fuzzy entropy}

Entropy is a general concept, which is used to measure the uncertainty of one system or a piece of information. Fuzzy degree is a quantitative index to describe the degree of fuzzy set. Fuzzy entropy is a method to measure the complexity of time series based on the concept of approximate entropy and sample entropy. The fuzzy entropy is described as follows [22]:

(1) Give an $N$ sample time series $\{u(i): 1 \leq i \leq N\}$. For given $m, n$ and $r$, a vector set $\left\{X_{i}^{m}, i=1,2, \ldots, N-m+1\right\}$ is formed. Each vector contains $m$ sequential elements starting from $u(i)$ as follow:

$X_{i}^{m}=\{u(i), u(i+1), \cdots, u(i+m-1)\}-u_{0}(i)$,

where $u_{0}(i)$ is the average of vector $X_{i}^{m}$ :

$u_{0}(i)=\frac{1}{m} \sum_{j=0}^{m-1} u(i+j)$

(2) For $X_{i}^{m}$, define the distance $d_{i j}^{m}$ between $X_{i}^{m}$ and $X_{j}^{m}(j=1,2,3, \ldots, N-m, i \neq j)$ as the maximum absolute difference of corresponding scalar components:

$d_{i j}^{m}=d\left[X_{i}^{m}, X_{j}^{m}\right]=\max _{k \in(0, m-1)}\left(u(i+k)-u_{0}(i)\right)-\left(u(j+k)-u_{0}(j)\right)$.

(3) Calculate the similarity degree $D_{i j}^{m}$ of $X_{j}^{m}$ to $X_{i}^{m}$ by using fuzzy function $\mu\left(d_{i j}^{m}, n, r\right)$ :

$D_{i j}^{m}(n, r)=\mu\left(d_{i j}^{m}, n, r\right)=e^{-\operatorname{In} 2\left(d_{i j}^{m} / r\right)^{n}}$.

(4) Define the function $\phi^{m}$ as follows:

$\phi^{m}(n, r)=\frac{1}{N-m} \sum_{i=1}^{N-m}\left(\frac{1}{N-m+!} \sum_{j=1, j \neq i}^{N-m} D_{i j}^{m}\right)$.

(5) Similarly, form $\left\{X_{i}^{m+1}\right\}$ and get the function $\phi^{m+1}$ :

$\phi^{m+1}(n, r)=\frac{1}{N-m} \sum_{i=1}^{N-m}\left(\frac{1}{N-m+!} \sum_{j=1, j \neq i}^{N-m} D_{i j}^{m+1}\right)$.

(6) Fuzzy entropy of sequence $\{u(i): 1 \leq i \leq N\}$ as the negative natural logarithm of the deviation of from is defined as follows:

$\operatorname{FuzzyEn}(m, n, r)=\lim _{N \rightarrow \infty}\left[\operatorname{In} \phi^{m}(n, r)-\operatorname{In} \phi^{m+1}(n, r)\right]$

(7) If the length $N$ is finite, $F u z z y E n(m, n, r)$ can be changed as follows:

$\operatorname{FuzzyEn}(m, n, r)=\operatorname{In} \phi^{m}(n, r)-\operatorname{In} \phi^{m+1}(n, r)$.

\subsection{Support vector machine}

The SVM is a supervised machine learning method based on structural risk minimization. It is 
to find one division plane to keep the point of training set far away the plane [23-27]. The kernel function in SVM is used to map the input space into the high-dimensional feature space. So, the selected kernel function ought to meet the following expression [28-31]:

$K\left(x_{i}, x_{j}\right)=\varphi\left(x_{i}\right)^{T} \bullet \varphi\left(x_{j}\right)$.

For the classification in different systems, the selected kernel functions are different. So, the radial basis function is selected for SVM. The kernel function is described:

$K\left(x, x_{i}\right)=\exp \left(-\gamma\left\|x-x_{i}\right\|^{2}\right)$,

where, $x$ is a $m$-dimension input vector, $x_{i}$ is the center of the $i$ th $\mathrm{RBF}$, and has the same dimension with $x, \gamma$ is the parameter of RBF kernel function.

\section{An improved PSO algorithm}

The basic PSO algorithm is a simple and practical algorithm, and its parameters are fixed. But it has weak local search ability and convergence speed and is easy to fall into local optimum solution. The values of parameters in the PSO determine the search performance, so the PSO algorithm is improved in order to improve the comprehensive performance.

\subsection{Improvement of learning factors}

\subsubsection{Linear change of learning factor}

In the general PSO algorithm, the $c_{1}$ and $c_{2}$ parameters are given in advance according to the experience. But their values $[0,4]$ will reduce the self learning ability of particles. In this paper, the value range of $c_{1}$ and $c_{2}$ parameters are given. The initial value and final value are $c_{1} \in(2.75,1.25)$ and $c_{2} \in(0.5,2.25)$, respectively. The learning factor function expression of linear change is descried:

$c_{1}=c_{1_{\text {max }}}+\left(c_{1_{\text {min }}}-c_{1_{\text {max }}}\right) \times t / T$,

$c_{2}=c_{2 \max }+\left(c_{2 \min }-c_{2 \max }\right) \times t / T$,

where $c_{1 \text { max }}$ and $c_{2 \text { max }}$ are the initial value of $c_{1}$ and $c_{2}, c_{1 \text { min }}$ and $c_{2 \min }$ are the final value of $c_{1}$ and $c_{2}, T$ is the maximum number of iterations, $t$ is the current number of iteration.

\subsubsection{Arctangent change of learning factor}

The study results are discovered that the PSO algorithm can make particles to leap the whole search space as much as possible, in order to obtain the diversity of particles in the initial stage of the search, and converge to the global optimal solution with fast speed in the end of the search under the ideal condition. By analyzing the influence of the change of learning factor, the arctangent function is used to dynamically adjust the $c_{1}$ and $c_{2}$ parameters in order to better balance the global search and the local search. The arctangent function expression is described:

$c_{1}=c_{1_{\text {max }}}-\left(c_{1_{\text {max }}}-c_{1_{\text {min }}}\right) \times(a \tan (20 \times t / T-e)+\operatorname{atan}(e)) / h$,

$c_{2}=c_{2 \max }-\left(c_{2 \max }-c_{2 \min }\right) \times(\operatorname{atan}(20 \times t / T-e)+a \tan (e)) / h$,

where $c_{1 \text { max }}$ and $c_{2 \text { max }}$ are the initial value of $c_{1}$ and $c_{2}, c_{1 \text { min }}$ and $c_{2 \min }$ are the final value of $c_{1}$ and $c_{2}, T$ is the maximum number of iterations, $t$ is the current number of iteration, and $e$ is the adjustment coefficient, $e=6 . h=\operatorname{atan}(20-e)+\operatorname{atan}(e)$. 


\subsection{Improvement of inertia weight}

The inertia weight $w$ is declined by using $S$ shape function in order to ensure that the population can maintain a high search speed in the initial search, decline the search speed in the middle search to easily converge to the global optimum, and keep a certain speed to finally converge to the optimal solution in the last search. The inertia weight expression of $S$ decreasing function is described:

$w=\left(w_{\max }-w_{\min }\right) /(1+\exp (2 \times o \times t / T-o))+w_{\min }$

where $w_{\max }$ and $w_{\min }$ are the maximum and minimum inertia weights. $w_{\max }=0.9$ and $w_{\min }=0.2$ are selected. $o$ is the control factor to adjust the speed, $o=13$ is set in here.

\subsection{Adaptive particle mutation strategy}

Because the $r_{1}$ and $r_{2}$ are random numbers on $(0,1)$, the selected different values will increase the randomness to update particle velocity and add the convergence time. In order to reduce the randomness, when the particles update their speed and position, if the $r_{1}$ or $r_{2}$ is more than 0.5 , then the self mutation on the $X_{i 1}^{k+1}$ is operated. The expressions are described:

$X_{i 1}^{k}=(20-1) \times r+1$,

$X_{i 2}^{k}=\left(g_{\max }-g_{\min }\right) \times r+g_{\min }$,

where $X_{i 1}^{k}$ and $X_{i 2}^{k}$ are the first dimension and the second dimension of $X$. That's to say, they are the changes of $C$ and $g$.

\section{Optimize the parameters of SVM}

\subsection{Optimization idea}

The parameters of kernel function are the key factors to affect the performance of SVM. For the RBF kernel function, the kernel parameter is the kernel width, which mainly affects the complexity of sample data in high dimensional feature space. The penalty parameter $C$ is used to adjust the confidence range of learning machine and proportion of empirical risk in the determined data subspace in order to obtain the best generalization ability. The values of $C$ is different in different data subspaces. In the determined data subspace, the value of $C$ represents the size of the empirical error penalty. There has at least one suitable $C$ in each data subspace to make the best generalization ability of SVM. If the value of $C$ is too larger, the corresponding penalty is too larger, the training error will become smaller and generalization ability will be poor. The early penalty factor and parameters in the kernel function need to be manually adjusted according to the training error. In recent years, the intelligent optimization algorithm is used to automatically adjust the values of penalty factors and the parameters of SVM. In this paper, the improved PSO algorithm is used to optimize the penalty factors and the parameters of the SVM in order to obtain the best SVM model with optimal classification effect.

\subsection{Optimization model and steps for SVM}

In this paper, the improved PSO algorithm is used to find the optimal combination of parameters for SVM. The optimization process is shown in Fig. 2.

\subsection{Analysis example}

In order to verify the validity of the improved PSO algorithm, the improved PSO(LWSPSO) 
algorithm by linear change of learning factor and $S$ decreasing function of inertia weight, and the improved PSO(AWAPSO) algorithm by arctangent change of learning factor, $S$ decreasing function of inertia weight and adaptive mutation strategy are compared in here. The PSO, LWSPSO and AWAPSO algorithms are used to optimize the SVM in here. The best fitness value and the average fitness value by using three algorithms are shown in Fig. 3-Fig. 5.

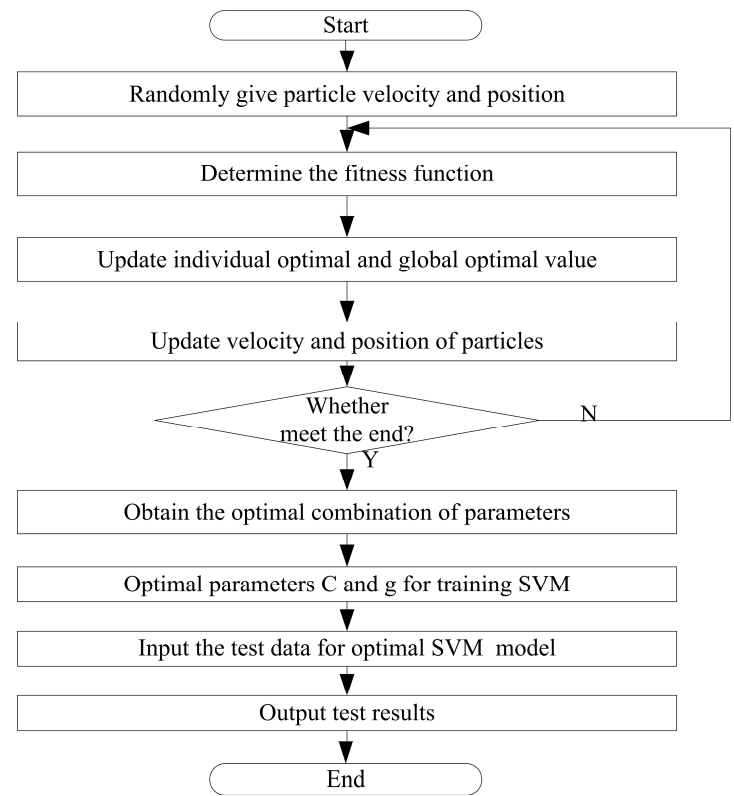

Fig. 2. The optimization process for SVM

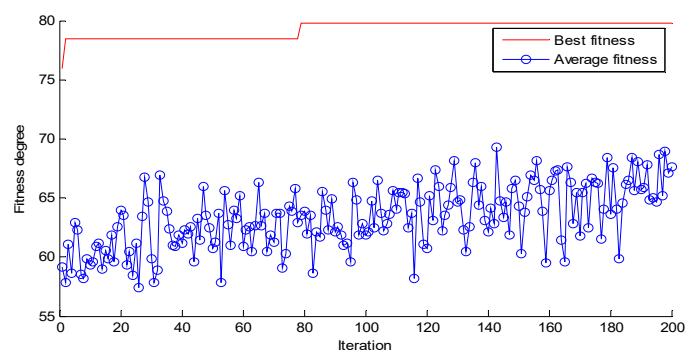

Fig. 3. The fitness values of PSO for optimizing SVM

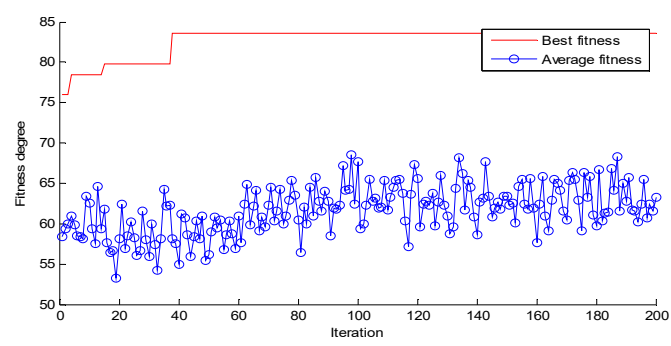

Fig. 4. The fitness values of LWSPSO for optimizing SVM

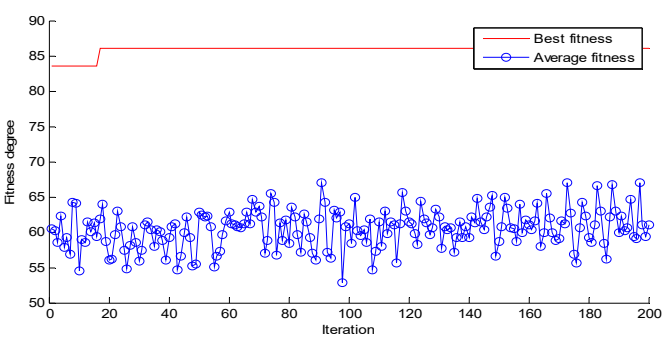

Fig. 5. The fitness values of AWAPSO for optimizing SVM

As can be seen from Fig. 3. to Fig. 5., with the increasing of the number of iterations, the best fitness values of three algorithms are changing. The PSO algorithm can obtain steadily fitness value at the 79th iteration, the LWSPSO algorithm can obtain steadily fitness value at the 39th 
iteration and the AWAPSO algorithm can obtain steadily fitness value at 17 th iteration. The experiment results show that the AWAPSO algorithm can find the optimal values of parameters and is the most accurate. The average fitness value of the AWAPSO algorithm is largest value in the early period. It can guarantee that the AWAPSO algorithm has a good global search ability. The AWAPSO algorithm can quickly converge to the global optimum value in the latter.

\section{A new fault diagnosis method}

\subsection{The idea of new fault diagnosis method}

The effective diagnosis method can ensure the safety and reliable operation of motor. In order to improve the diagnosis accuracy for motor bearing fault, a new fault diagnosis method based on integrating EMD, fuzzy entropy, improved PSO algorithm and SVM is proposed in this paper. Firstly, the EMD method based on the direct extraction of the energy associated with various intrinsic time scales is used to decompose the vibration signals of motor bearing into a series of intrinsic mode functions(IMFs) and residual signal. The IMFs are only used in this paper. Then, the fuzzy entropy with measuring the complexity of time series based on the concept of approximate entropy and sample entropy is used to effectively extract the feature of vibration signal, which is regarded as input vectors. The dynamic adjustment strategy of learning factor, decreasing inertia weight of function and adaptive mutation strategy of particle are used to improve the PSO algorithm in order to improve the optimization ability of PSO algorithm, which is used to optimize the parameters of SVM model for improving the classification accuracy. Finally, a new fault diagnosis method is proposed in order to realize the fault diagnosis of motor bearing and obtain diagnosis results.

\subsection{The fault diagnosis model and steps}

The new fault diagnosis model of motor bearing based on combining EMD, fuzzy entropy, improved PSO algorithm and SVM is constructed. And the flow of the new fault diagnosis model is shown in Fig. 6.

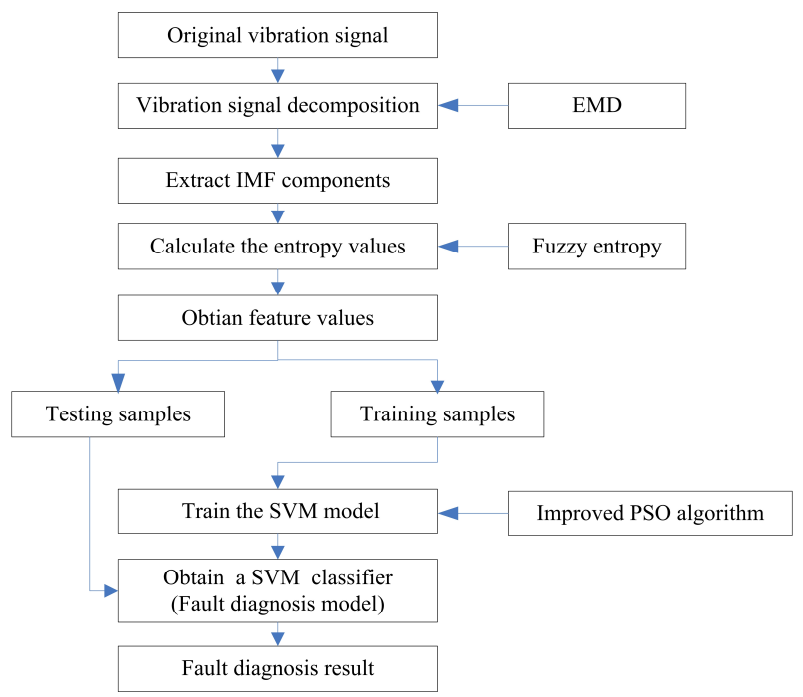

Fig. 6. The new fault diagnosis model for motor bearing

The steps are described as follows:

(1) The vibration signal under each state is decomposed by using EMD with adaptive decomposition capability in order to obtain a series of IMF components. 
(2) Calculate the fuzzy entropy values of IMFs by using fuzzy entropy technology in order to construct the feature vectors. Then the feature vectors are divided into training samples and testing samples in order to train the SVM model and test the fault diagnosis model.

(3) The learning factor, inertia weight and adaptive particle mutation in the PSO algorithm are improved in obtain the improved PSO algorithm with the higher optimization performance for solving complex optimization problem.

(4) The improved PSO algorithm is used to optimize the kernel parameter $\sigma^{2}$ and penalty coefficient $C$ of SVM model.

(5) The training samples are used to train the SVM in order to obtain optimal SVM classifier (fault diagnosis model).

(6) The testing samples are used to test the obtained fault diagnosis model.

(7) Obtain the diagnosis result.

\section{Fault diagnosis case and result analysis}

\subsection{Experimental environment and data}

In order to validate the effectiveness of the proposed fault diagnosis method, the vibration data from Bearing Data Center of Case Western Reserve University is selected in this paper [32]. The 6205-2RS 6 JEM SKF deep groove ball bearing is employed in the experiment. The motor is connected to a dynamometer and torque sensor by a self-aligning coupling. The data were collected from an accelerometer mounted on the motor housing at the drive end of the motor. The vibration signals were measured under 0 -load $(0 \mathrm{hp})$ at rotation speed of $1797 \mathrm{r} / \mathrm{min}$. Faults were introduced to the test bearings by using electro-discharge machining method. The fault diameter was 0.007 ". Four different operating conditions are:(1) normal condition; (2) inner race fault; (3) outer race fault; and (4) rolling element fault. The bearing vibration data was sampled at the frequency of $12000 \mathrm{~Hz}$ and the duration of each vibration signal was 10 seconds. The original data were divided into the segments that each segment covered 4096 data points.

\subsection{Case analysis for fault diagnosis}

\subsubsection{Vibration data decomposition}

According to the definition of EMD method and the steps of signal processing, the EMD method is firstly used to decompose the high frequency of original vibration signal, then the low frequency and relatively low frequency of original vibration signal are decomposed. That's to say, its decomposition for the original vibration signal is regular. So, the frequency components of IMF components are different. And for different vibration signal, the EMD method takes on adaptive decomposition capability, and the results of the decomposition are unique. In general, the EMD method decomposes the he original vibration signal, rolling element fault vibration signal, fault inner ring vibration signal and the outer ring fault vibration signal into a series of IMF components with the primary information of the original vibration signal. The decomposition results are shown in Fig. 7-Fig. 10.

\subsubsection{Fault feature extraction based on Fuzzy Entropy}

In the fuzzy entropy, it has a great effect on the entropy calculation result for selecting parameters. If the value of model dimension $m$ is larger, it can better reflect the dynamic evolution process of signal. If the similar tolerance $r$ is larger, it will assembly increase the information loss. If the similar tolerance $r$ is smaller, it will be sensitive to the noise and lead to indefinitely increase entropy value. Based on comprehensive analysis, the parameters of fuzzy entropy are set as follows: $m=2, r=0.15 S D$. 


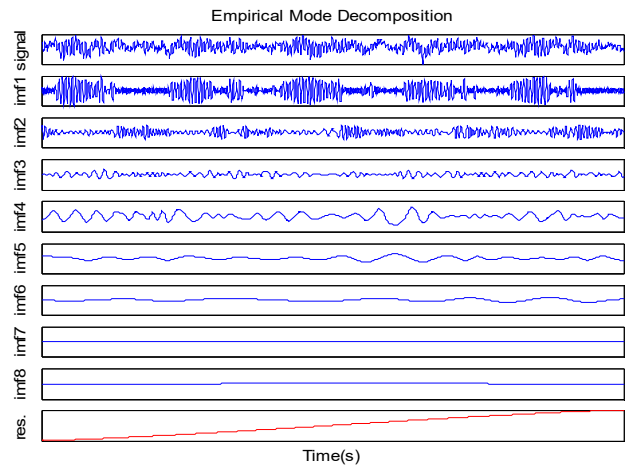

Fig. 7. Decomposition result of normal signal

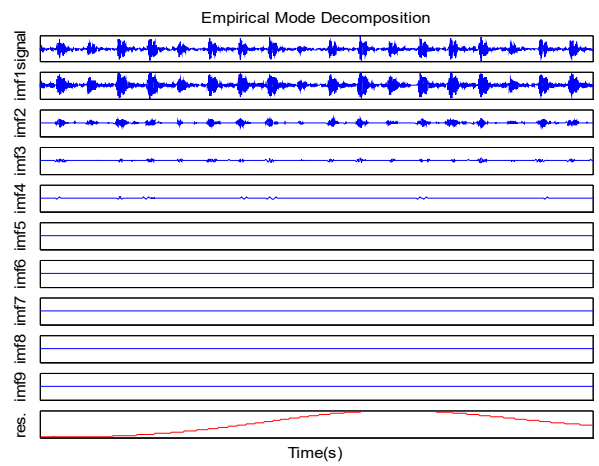

Fig. 9. Decomposition result of outer ring signal

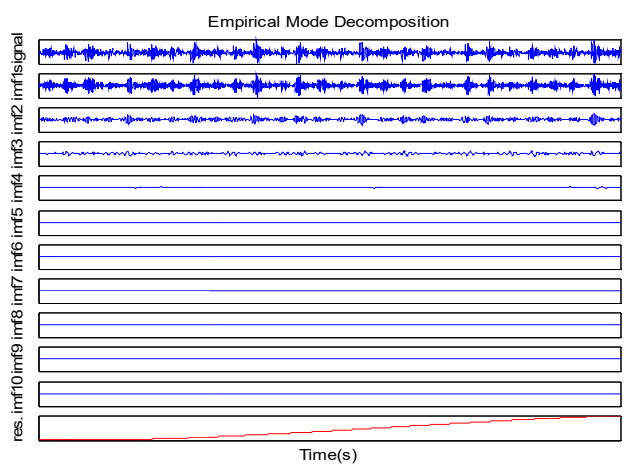

Fig. 8. Decomposition result of inner ring signal

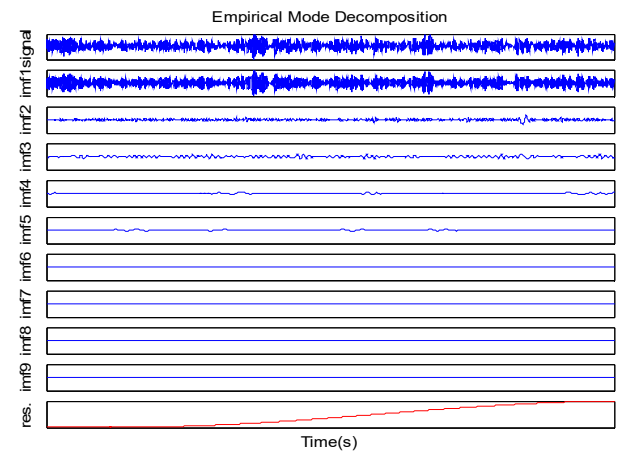

Fig. 10. Decomposition result of rolling element signal

The effective values of parameters are set in order to obtain the feature value of vibration signal by using fuzzy entropy technology. The fuzzy entropy values for the normal vibration signal, rolling element fault vibration signal, inner ring fault vibration signal and outer ring fault vibration signal are show in Table 1-Table 4. Each condition obtains 20 sets of fuzzy entropy values.

Table 1. Fuzzy entropy values of the normal vibration signal

\begin{tabular}{|c|c|c|c|c|c|c|c|c|c|c|}
\hline M1 & M2 & M3 & M4 & M5 & M6 & M7 & M8 & M9 & M10 & M11 \\
\hline 3.3735 & 3.0103 & 0.2427 & 0.0737 & 0.0067 & 0.0005 & -0.0005 & -0.0005 & -0.0005 & 0 & 0 \\
\hline 5.8110 & 4.2864 & 0.3589 & 0.0841 & 0.0171 & 0.0010 & -0.0003 & -0.0005 & -0.0005 & 0 & 0 \\
\hline 1.2732 & 3.9607 & 0.2636 & 0.0455 & 0.0100 & 0.000 & -0.0004 & -0.0005 & 0 & 0 & 0 \\
\hline 11.1880 & 4.4976 & 0.2081 & 0.0473 & 0.0050 & 0.0012 & -0.0003 & -0.0005 & -0.0005 & 0 & 0 \\
\hline
\end{tabular}

Table 2. Fuzzy entropy values of the fault vibration signal of rolling element

\begin{tabular}{|c|c|c|c|c|c|c|c|c|c|c|}
\hline M1 & M2 & M3 & M4 & M5 & M6 & M7 & M8 & M9 & M10 & M11 \\
\hline 25.7745 & 3.3235 & 0.85 & 0.0953 & 0.0192 & 0.0015 & -0.0002 & -0.0005 & -0.0005 & -0.0005 & 0 \\
\hline 38.7103 & 3.7037 & 1.1101 & 0.0856 & 0.0197 & 0.0007 & -0.0003 & -0.0005 & -0.0005 & -0.0005 & 0 \\
\hline 16.1817 & 8.7728 & 1.1915 & 0.2188 & 0.0237 & 0.0012 & -0.0002 & -0.0005 & -0.0005 & -0.0005 & 0 \\
\hline 32.0226 & 8.2626 & 1.9099 & 0.1084 & 0.0177 & 0.0012 & -0.0003 & -0.0005 & -0.0005 & 0 & 0 \\
\hline
\end{tabular}

Table 3. Fuzzy entropy values of the fault vibration signal of inner ring

\begin{tabular}{|c|c|c|c|c|c|c|c|c|c|c|}
\hline M1 & M2 & M3 & M4 & M5 & M6 & M7 & M8 & M9 & M10 & M11 \\
\hline 55.6789 & 21.3713 & 6.0573 & 0.5262 & 0.0618 & 0.0095 & 0.001 & -0.0003 & -0.0005 & -0.0005 & -0.0005 \\
\hline 104.5150 & 55.7308 & 13.6520 & 0.9935 & 0.0799 & 0.0089 & 0.0011 & -0.0004 & -0.0005 & -0.0005 & 0 \\
\hline 75.8021 & 58.1719 & 31.2462 & 2.1151 & 0.1071 & 0.0238 & 0.0024 & -0.0002 & -0.0004 & -0.0005 & -0.0005 \\
\hline 98.1662 & 23.7256 & 10.1012 & 1.3721 & 0.0586 & 0.0117 & 0.0007 & -0.0003 & -0.0005 & -0.0005 & 0 \\
\hline
\end{tabular}


Table 4. Fuzzy entropy values of the fault vibration signal of outer ring

\begin{tabular}{|c|c|c|c|c|c|c|c|c|c|c|}
\hline M1 & M2 & M3 & M4 & M5 & M6 & M7 & M8 & M9 & M10 & M11 \\
\hline 34.0079 & 55.24 & 2.189 & 0.6729 & 0.0678 & 0.0049 & 0.0008 & -0.0004 & -0.0005 & -0.0005 & 0 \\
\hline 62.4249 & 23.5713 & 1.9605 & 1.7625 & 0.0484 & 0.0014 & -0.0001 & -0.0004 & -0.0005 & 0 & 0 \\
\hline 51.3327 & 68.2587 & 3.6419 & 0.453 & 0.0514 & 0.0056 & 0.0002 & -0.0003 & -0.0005 & -0.0005 & -0.0005 \\
\hline 21.906 & 44.1365 & 3.6347 & 0.2234 & 0.0434 & 0.0034 & 0 & -0.0004 & -0.0005 & -0.0005 & 0 \\
\hline
\end{tabular}

\subsubsection{Fault diagnosis result}

Due to the small sample and high dimension of the fault diagnosis for motor bearing, the support vector machine (SVM) is selected as a classifier. The obtained fuzzy entropy values are selected as feature vectors, which are input into the SVM model in order to obtain the SVM classifier. The data set consists of 80 data samples of four conditions (normal condition, outer race fault, inner race fault and rolling element fault) under 0-load. Each of the four conditions includes 20 data samples. There are 40 samples for training and 40 for testing. Because of four different vibration signals, it is necessary to construct 3 two-classifiers, shown in Fig. 11.

Due to the random search algorithm of the PSO, the average value of 10 times is regarded as the final classification accuracy. The diagnosis results by the PSO and SVM (PSO-SVM) is obtained in Table 5. The fault diagnosis results by the LWSPSO algorithm and SVM (LWSPSO-SVM) is obtained in Table 6. The fault diagnosis results by the AWAPSO algorithm and SVM(AWAPSO-SVM) is obtained in Table 7. The diagnosis accuracy comparison for four methods is shown in Fig. 12.

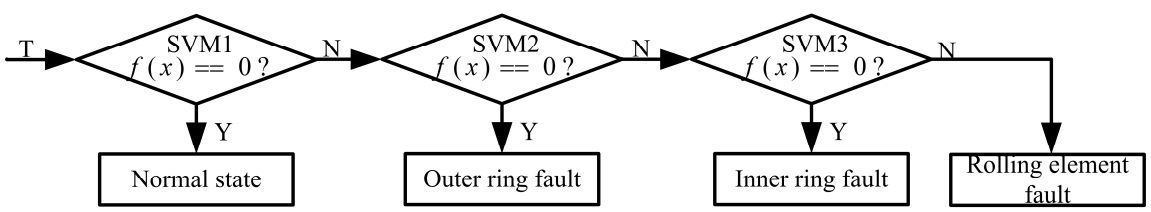

Fig. 11. Multi-classifier based on SVM

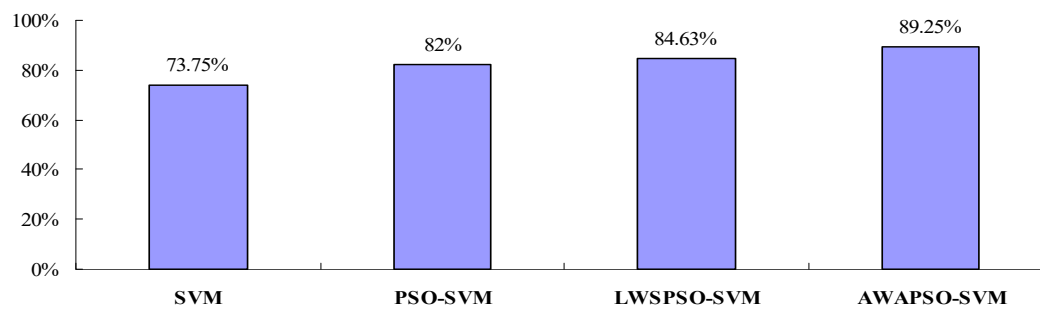

Fig. 12. The diagnosis accuracy comparison for four methods

Table 5. The diagnosis results by PSO-SVM

\begin{tabular}{|c|c|c|c|c|c|c|c|c|c|c|c|}
\hline Time & 1 & 2 & 3 & 4 & 5 & 6 & 7 & 8 & 9 & 10 & AVG \\
\hline $\begin{array}{c}\text { Accuracy } \\
\text { rate (\%) }\end{array}$ & 75.00 & 87.5 & 88.75 & 90.00 & 76.25 & 81.25 & 78.75 & 73.75 & 81.25 & 87.50 & 82.00 \\
\hline$c_{1}$ & 2.00 & 2.00 & 2.00 & 2.00 & 2.00 & 2.00 & 2.00 & 2.00 & 2.00 & 2.00 & \\
\hline$c_{2}$ & 2.00 & 2.00 & 2.00 & 2.00 & 2.00 & 2.00 & 2.00 & 2.00 & 2.00 & 2.00 & \\
\hline$C$ & 86.88 & 51.03 & 14.20 & 24.79 & 50.84 & 91.98 & 24.39 & 90.33 & 71.29 & 91.57 & \\
\hline$g$ & 80.71 & 13.90 & 13.52 & 25.09 & 70.04 & 53.28 & 67.47 & 88.33 & 53.63 & 27.84 & \\
\hline Iteration & 1 & 1 & 1 & 8 & 1 & 1 & 1 & 1 & 1 & 1 & 1.70 \\
\hline $\begin{array}{c}\text { Running } \\
\text { time }\end{array}$ & 35.11 & 31.76 & 30.83 & 36.46 & 33.83 & 31.44 & 31.23 & 31.62 & 35.77 & 34.98 & 33.30 \\
\hline
\end{tabular}

As can be seen from Table 5, for the PSO-SVM method, the best diagnosis accuracy rate and the average diagnosis accuracy rate are $90 \%$ and $82 \%$, respectively. the smallest iteration and the 
average iteration are 1 and 1.7 iterations, respectively. The least running times and the average running times are $30.83 \mathrm{~s}$ and $33.3 \mathrm{~s}$. As can be seen from Table 6, for the LWSPSO-SVM method, the best diagnosis accuracy rate and the average diagnosis accuracy rate are $90 \%$ and $84.63 \%$, respectively. the smallest iteration and the average iteration are 1 and 11.7 iterations, respectively. The least running times and the average running times are $25.54 \mathrm{~s}$ and $25.94 \mathrm{~s}$. As can be seen from Table 7, for the AWAPSO-SVM method, the best diagnosis accuracy rate and the average diagnosis accuracy rate are $90 \%$ and $89.25 \%$, respectively. The smallest iteration and the average iteration are 1 and 43.2 iterations, respectively. The least running times and the average running times are $25.94 \mathrm{~s}$ and $28.23 \mathrm{~s}$. By analyzing the diagnosis results from Table 5 to Table 8 , the PSO-SVM, LWSPSO-SVM and AWAPSO-SVM methods can obtain the best diagnosis accuracy rate of $90 \%$. But the average diagnosis accuracy rate of $73.75 \%$ for the SVM method is lowest, and the average diagnosis accuracy rate of $89.25 \%$ for the AWAPSO-SVM method is highest.

Table 6. The diagnosis results by LWSPSO-SVM

\begin{tabular}{|c|c|c|c|c|c|c|c|c|c|c|c|}
\hline Times & 1 & 2 & 3 & 4 & 5 & 6 & 7 & 8 & 9 & 10 & AVG \\
\hline $\begin{array}{c}\text { Accuracy } \\
\text { rate (\%) }\end{array}$ & 90.00 & 87.5 & 81.25 & 86.25 & 81.25 & 80.00 & 87.50 & 87.50 & 83.75 & 81.25 & 84.63 \\
\hline$c_{1}$ & 2.74 & 2.74 & 2.74 & 2.74 & 2.74 & 2.62 & 2.14 & 2.67 & 2.74 & 2.74 & \\
\hline$c_{2}$ & 0.51 & 0.51 & 0.51 & 0.51 & 0.51 & 0.65 & 1.21 & 0.60 & 0.52 & 0.51 & \\
\hline$w$ & 0.90 & 0.90 & 0.90 & 0.90 & 0.90 & 0.90 & 0.85 & 0.90 & 0.90 & 0.90 & \\
\hline$C$ & 37.89 & 81.07 & 62.77 & 81.97 & 78.82 & 54.80 & 23.61 & 69.96 & 94.36 & 53.50 & \\
\hline$g$ & 19.75 & 15.35 & 62.08 & 37.29 & 59.01 & 0.01 & 9.46 & 12.71 & 43.93 & 58.58 & \\
\hline Iteration & 1 & 1 & 1 & 1 & 1 & 17 & 81 & 11 & 2 & 1 & 11.70 \\
\hline $\begin{array}{c}\text { Running } \\
\text { time }\end{array}$ & 25.54 & 26.47 & 25.82 & 25.97 & 25.66 & 25.94 & 26.13 & 26.28 & 25.72 & 25.91 & 25.94 \\
\hline
\end{tabular}

Table 7. The diagnosis results by AWAPSO-SVM

\begin{tabular}{|c|c|c|c|c|c|c|c|c|c|c|c|}
\hline Time & 1 & 2 & 3 & 4 & 5 & 6 & 7 & 8 & 9 & 10 & AVG \\
\hline $\begin{array}{c}\text { Accuracy } \\
\text { rate (\%) }\end{array}$ & 88.75 & 90.00 & 90.00 & 90.00 & 87.50 & 90.00 & 88.75 & 87.50 & 90.00 & 90.00 & 89.25 \\
\hline$c_{1}$ & 1.26 & 1.28 & 2.75 & 2.75 & 2.75 & 1.87 & 2.75 & 2.57 & 2.75 & 2.75 & \\
\hline$c_{2}$ & 2.24 & 2.22 & 0.50 & 0.50 & 0.50 & 1.52 & 0.50 & 0.71 & 0.50 & 0.50 & \\
\hline$w$ & 0.20 & 0.205 & 0.90 & 0.90 & 0.90 & 0.90 & 0.90 & 0.90 & 0.90 & 0.90 & \\
\hline$C$ & 14.54 & 100 & 82.21 & 68.67 & 66.24 & 46.51 & 39.07 & 85.35 & 52.46 & 55.55 & \\
\hline$g$ & 11.92 & 24.84 & 18.49 & 20.87 & 13.36 & 22.10 & 25.30 & 8.60 & 18.70 & 17.51 & \\
\hline Iteration & 182 & 138 & 1 & 1 & 2 & 63 & 1 & 42 & 1 & 1 & 43.20 \\
\hline $\begin{array}{c}\text { Running } \\
\text { time }\end{array}$ & 27.88 & 25.94 & 28.78 & 26.76 & 26.92 & 30.88 & 27.37 & 30.38 & 30.57 & 26.83 & 28.23 \\
\hline
\end{tabular}

The average diagnosis accuracy rate of the AWAPSO-SVM method is improved $7.25 \%$ than the average diagnosis accuracy rate of the PSO-SVM method. The average diagnosis accuracy rate of the AWAPSO-SVM method is best among four fault diagnosis methods. This result show that the proposed AWAPSO algorithm takes on better optimization performance, and can obtain the better combination values of parameters of SVM to construct a better fault diagnosis model with the higher and stable classification result. For the running time of algorithms, the average running time of $0.01 \mathrm{~s}$ for SVM is least, and the average running time of $33.3 \mathrm{~s}$ for PSO-SVM is longest. And the average running time of AWAPSO-SVM method is $28.23 \mathrm{~s}$. The running time of LWSPSO-SVM is least among PSO-SVM, LWSPSO-SVM and AWAPSO-SVM methods. In general, the proposed AWAPSO-SVM method takes on the lower complexity, stronger randomness and better optimization performance. And the fault diagnosis method based on AWAPSO-SVM takes on good classification result and can quickly diagnose the motor bearing faults. 
Table 8. The accuracy and running time of four methods

\begin{tabular}{|c|c|c|c|c|c|c|c|c|c|c|c|c|}
\hline $\begin{array}{c}\text { Diagnosis } \\
\text { method }\end{array}$ & Indexes & 1 & 2 & 3 & 4 & 5 & 6 & 7 & 8 & 9 & 10 & AVG \\
\hline \multirow{2}{*}{ SVM } & $\begin{array}{c}\text { Accuracy } \\
\text { rate }(\%)\end{array}$ & 73.75 & 73.75 & 73.75 & 73.75 & 73.75 & 73.75 & 73.75 & 73.75 & 73.75 & 73.75 & 73.75 \\
\hline & $\begin{array}{c}\text { Running } \\
\text { time }\end{array}$ & 0.01 & 0.01 & 0.01 & 0.01 & 0.01 & 0.01 & 0.01 & 0.01 & 0.01 & 0.01 & 0.01 \\
\hline \multirow{2}{*}{ PSO-SVM } & $\begin{array}{c}\text { Accuracy } \\
\text { rate }(\%)\end{array}$ & 5.00 & 87.50 & 8.75 & 90.00 & 76.25 & 81.25 & 78.75 & 73.75 & 81.25 & 87.50 & 82.00 \\
\hline & $\begin{array}{c}\text { Running } \\
\text { time }\end{array}$ & 35.11 & 31.76 & 30.83 & 36.46 & 33.83 & 31.44 & 31.23 & 31.62 & 35.77 & 34.98 & 33.30 \\
\hline \multirow{2}{*}{$\begin{array}{c}\text { LWSPSO } \\
\text {-SVM }\end{array}$} & $\begin{array}{l}\text { Accuracy } \\
\text { rate }(\%)\end{array}$ & 90.00 & 87.5 & 81.25 & 86.25 & 81.25 & 80.00 & 87.50 & 87.50 & 83.75 & 81.25 & 84.63 \\
\hline & $\begin{array}{c}\text { Running } \\
\text { time }\end{array}$ & 5.54 & 26.47 & 5.82 & 25.97 & 25.66 & 25.94 & 26.13 & 26.28 & 25.72 & 25.91 & 25.94 \\
\hline \multirow{2}{*}{$\begin{array}{c}\text { AWAPSO } \\
\text {-SVM }\end{array}$} & $\begin{array}{c}\text { Accuracy } \\
\text { rate }(\%)\end{array}$ & 88.75 & 90.00 & 90.00 & 90.00 & 87.50 & 90.00 & 88.75 & 87.50 & 90.00 & 90.00 & 89.25 \\
\hline & $\begin{array}{c}\text { Running } \\
\text { time }\end{array}$ & 27.88 & 25.94 & 28.78 & 26.76 & 26.92 & 30.88 & 27.37 & 30.38 & 30.57 & 26.83 & 28.23 \\
\hline
\end{tabular}

Wu Deng conceived the research subject and contributed to feature extraction, Rui Yao contributed to vibration signal decomposition, Mneg Sun contributed to value calculation of fuzzy entropy, Huimin Zhao contributed to optimization of support vector machine, Yinglian Luo contributed to improved particle swarm optimization algorithm, Chang Dong carried out the experiments. All authors have read and approved the final manuscript.

\section{Conclusions}

To effectively extract the features of fault vibration signal and well diagnose the faults of motor bearing, a novel fault diagnosis method based on integrating EMD, fuzzy entropy, improved PSO algorithm and SVM is proposed in this paper. In the proposed fault diagnosis method, the EMD method is used to decompose the vibration signals of motor bearing into a series of intrinsic mode functions (IMFs). The fuzzy entropy is used to effectively extract the feature of vibration signal, which is regarded as input vectors. Then the dynamic adjustment strategy of learning factor, decreasing inertia weight of function and adaptive mutation strategy of particles are introduced into the basic PSO algorithm to obtain an improved PSO algorithm, which is used to optimize the parameters of penalty function and kernel function of SVM in order to obtain an effective fault diagnosis model with the higher accuracy. Finally, the actual vibration signal of motor bearing is used to validate the effectiveness of the proposed fault diagnosis method. In the experiment result, the average diagnosis accuracy rates of the SVM classifier, the PSO-SVM classifier, the LWSPSO-SVM classifier and the AWAPSO-SVM classifier are $73.75 \%, 82 \%, 84.63 \%$ and $89.25 \%$, respectively. And the average running times of the PSO-SVM classifier, the LWSPSO-SVM classifier and the AWAPSO-SVM classifier are very nearly the same. So, the experiment results show that the improved PSO algorithm takes on the faster convergence speed, faster convergence accuracy and better optimization performance. And the proposed fault diagnosis method can effectively obtain the diagnosis result. It can quickly diagnose the motor bearing faults with the higher accuracy and reliability.

\section{Acknowledgements}

The authors would like to thank all the reviewers for their constructive comments. This research was supported by the National Natural Science Foundation of China (51475065, 51605068, U1433124), Open Project Program of State Key Laboratory of Mechanical Transmissions (Chongqing University) (SKLMT-KFKT-201513), the Natural Science 
Foundation of Liaoning Province (2015020013), the Open Project Program of Sichuan Provincial Key Lab of Process Equipment and Control (GK201613),Open Project Program of the Traction Power State Key Laboratory of Southwest Jiaotong University (TPL1705).

\section{References}

[1] Wang S. B., Cai G. G., Zhu Z. K., Huang W. G., Zhang X. W. Transient signal analysis based on Levenberg-Marquardt method for fault feature extraction of rotating machines. Mechanical Systems and Signal Processing, Vol. 54, Issue 55, 2015, p. 16-40.

[2] Xue Y., Jiang J. M., Zhao B. P., Ma T. H. A self-adaptive artificial bee colony algorithm based on global best for global optimization. Soft Computing, 2017, https://doi.org/10.1007/s00500-017-2547-1.

[3] van Wyk B. J., van Wyk M. A., Qi G. Difference histograms: a new tool for time series analysis applied to bearing fault diagnosis. Pattern Recognition Letters, Vol. 30, Issue 6, 2009, p. 595-599.

[4] Chen J., Patton R. J., Liu G. Optimal residual design for fault diagnosis using multi-objective optimization and genetic algorithms. International Journal of Systems Science, Vol. 27, Issue 6, 1996, p. 567-576.

[5] Wang L., Niu Q., Fei M. R. A novel quantum ant colony optimization algorithm and its application to fault diagnosis. Transactions of the Institute of Measurement and Control, Vol. 30, Issues 3-4, 2008, p. 313-329.

[6] Zhang X. L., Chen X. F., He Z. J. Fault diagnosis based on support vector machines with parameter optimization by an ant colony algorithm. Proceedings of the Institution of Mechanical Engineers, Part C: Journal of Mechanical Engineering Science, Vol. 224, Issue 1, 2010, p. 217-229.

[7] Zhang L., Xiong G., Liu H., Zou H., Guo W. Fault diagnosis based on optimized node entropy using lifting wavelet packet transform and genetic algorithms. Proceedings of the Institution of Mechanical Engineers. Part I: Journal of Systems and Control Engineering, Vol. 224, Issue 5, 2010, p. 557-573.

[8] Zhao C. L., Sun X. B., Sun S. L., Jiang T. Fault diagnosis of sensor by chaos particle swarm optimization algorithm and support vector machine. Expert Systems with Applications, Vol. 38, Issue 8, 2011, p. 9908-9912.

[9] Azade A., Saberi M., Kazem A., Ebrahimipour V., Nourmohammadzadeh A., Saberi Z. A flexible algorithm for fault diagnosis in a centrifugal pump with corrupted data and noise based on ANN and support vector machine with hyper-parameters optimization. Applied Soft Computing Journal, Vol. 13, Issue 3, 2013, p. 1478-1485.

[10] Du W. L., Li A. S., Ye P. F., Liu C. L. Fault diagnosis of plunger pump in truck crane based on relevance vector machine with particle swarm optimization algorithm. Shock and Vibration, Vol. 20, Issue 4, 2013, p. 781-792.

[11] Zhu K. H., Song X. G., Xue D. X. A roller bearing fault diagnosis method based on hierarchical entropy and support vector machine with particle swarm optimization algorithm. Measurement: Journal of the International Measurement Confederation, Vol. 47, Issue 1, 2014, p. 669-675.

[12] Unal M., Onat M., Demetgul M., Kucuk H. Fault diagnosis of rolling bearings using a genetic algorithm optimized neural network. Measurement: Journal of the International Measurement Confederation, Vol. 58, 2014, p. 187-196.

[13] Ao H., Cheng J. S., Yang Y., Truong T. K. The support vector machine parameter optimization method based on artificial chemical reaction optimization algorithm and its application to roller bearing fault diagnosis. Journal of Vibration and Control, Vol. 21, Issue 12, 2015, p. 2434-2445.

[14] Zhang X. L., Chen W., Wang B. J., Chen X. F. Intelligent fault diagnosis of rotating machinery using support vector machine with ant colony algorithm for synchronous feature selection and parameter optimization. Neurocomputing, Vol. 167, 2015, p. 260-279.

[15] Yang D.L., Liu Y.L., Li S.B., Li X.J., Ma L.Y. Gear fault diagnosis based on support vector machine optimized by artificial bee colony algorithm. Mechanism and Machine Theory, Vol. 90, 2015, p. 219-229.

[16] Chu D. L., He Q., Mao X. Rolling bearing fault diagnosis by a novel fruit fly optimization algorithm optimized support vector machine. Journal of Vibroengineering, Vol. 18, Issue 1, 2016, p. 151-164.

[17] Duan L. X., Xie M. Y., Bai T. B., Wang J. J. A new support vector data description method for machinery fault diagnosis with unbalanced datasets. Expert Systems with Applications, Vol. 64, 2016, p. 239-246. 
[18] Su Z. Q., Tang B. P., Liu Z. R., Qin Y. Multi-fault diagnosis for rotating machinery based on orthogonal supervised linear local tangent space alignment and least square support vector machine. Neurocomputing, Vol. 157, 2015, p. 208-222.

[19] Zhang X. Y., Qiu D. Y., Chen F. Support vector machine with parameter optimization by a novel hybrid method and its application to fault diagnosis. Neurocomputing, Vol. 149, 2015, p. 641-651.

[20] Kennedy J., Eberhart R. Particle swarm optimization. Proceedings of the IEEE International Conference on Neural Networks, 1995, p. 1942-1948.

[21] Yu D. J., Cheng J. S., Yang Y. Application of EMD method and Hilbert spectrum to the fault diagnosis of roller bearings. Mechanical Systems and Signal Processing, Vol. 19, Issue 2, 2005, p. 259-270.

[22] Li Y. B., Xu M. Q., Zhao H. Y., Huang W. H. Hierarchical fuzzy entropy and improved support vector machine based binary tree approach for rolling bearing fault diagnosis. Mechanism and Machine Theory, Vol. 98, 2016, p. 114-132.

[23] Vapnik V. The Nature of Statistical Learning Theory. Springer Verlag, New York, 1995.

[24] Gu B., Sheng V. S., Tay K. Y., Romano W., Li S. Incremental support vector learning for ordinal regression. IEEE Transactions on Neural Networks and Learning Systems, Vol. 26, Issue 7, 2015, p. 1403-1416.

[25] Gu B., Sheng V. S. A robust regularization path algorithm for $v$-support vector classification. IEEE Transactions on Neural Networks and Learning Systems, 2016, https://doi.org/10.1109/TNNLS.2016.2527796.

[26] Wen X. Z., Shao L., Xue Y., Fang W. A rapid learning algorithm for vehicle classification. Information Sciences, Vol. 295, Issue 1, 2015, p. 395-406.

[27] Gu B., Sun X. M., Sheng V. S. Structural minimax probability machine. IEEE Transactions on Neural Networks and Learning Systems, 2016, https://doi.org/10.1109/TNNLS.2016.2544779.

[28] Gu B., Sheng V. S., Wang Z. J., Ho D., Osman S., Li S. Incremental learning for v-support vector regression. Neural Networks, Vol. 67, 2015, p. 140-150.

[29] Xia Z. H., Wang X. H., Sun X. M., Wang B. W. Steganalysis of least significant bit matching using multi-order differences. Security and Communication Networks, Vol. 7, Issue 8, 2014, p. 283-1291.

[30] Zheng Y. H., Jeon B., Xu D. H., Wu Q. M. J., Zhang H. Image segmentation by generalized hierarchical fuzzy C-means algorithm. Journal of Intelligent and Fuzzy Systems, Vol. 28, Issue 2, 2015, p. 961-973.

[31] Case Western Reserve University Bearing Data Center Website. http://www.eecs.case.

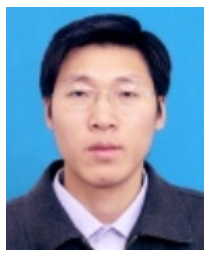

Wu Deng, Professor, received the Doctor degree in computer science and technology from Dalian Maritime University in 2012. The research interests: artificial intelligence, fault diagnosis.

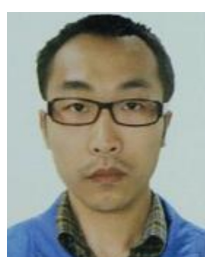

Rui Yao, graduate students at the school of electrical and information, Dalian Jiaotong University. The research interests: artificial intelligence, fault diagnosis.

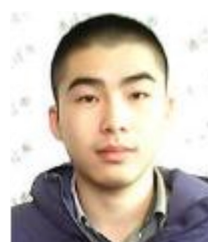

Meng Sun, graduate students at Software Institute, Dalian Jiaotong University. The research interests: artificial intelligence, optimization. 


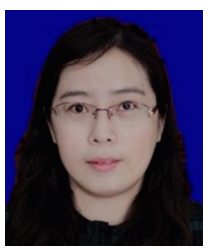

Huimin Zhao, Associate Professor, received the Doctor degree in mechanical engineering and automation from Dalian Jiaotong University in 2013. The research interests: artificial intelligence, signal processing, fault diagnosis.

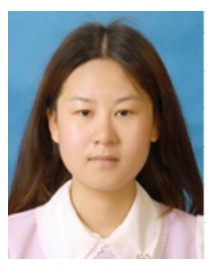

Yinglian Luo, graduate students at Software Institute, Dalian Jiaotong University. The research interests: artificial intelligence, fault diagnosis.

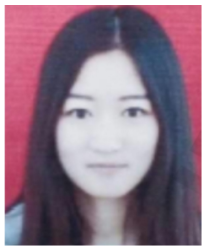

Chang Dong, graduate students at Software Institute, Dalian Jiaotong University. The research interests: artificial intelligence, fault diagnosis. 